\title{
Vernal Keratoconjunctivitis and its Management Challenges
}

Dr. Onyeahiri Collins, OD, MNOA, Ophthalmology Department Abubakar Tafawa Balewa University Teaching Hospital, Bauchi, P.M.B.0117 Bauchi, Bauchi State

Correspondence may directed to: omacoll@yahoo.com

\begin{abstract}
AbstractVernal keratoconjunctivitis (VKC) is an allergic, bilateral, recurrent inflammatory disorder of the conjunctiva and the cornea that has a seasonal incidence. It affects young males more than females in age bracket of 3 to 16 . In the majority of cases, symptoms resolve at puberty, but some may run into adulthood. Diagnosis is based typically on clinical signs and symptoms. The cause of the disease is not clearly known, but it is often associated with atopic diseases such as asthma or eczema and is probably due to a longstanding allergic reaction.

The disease consists clinically of the palpebral, limbal and mixed types. Its management has been a great challenge to eye care providers because of its recurrent nature, the large number of individuals who are affected, wrong diagnoses, and lack of judicious drug administration. The disease has the potential of producing serious vision-threatening complications if not adequately managed. This case report also reviews the diagnosis and management options for patients with mixed VKC and demonstrates the importance of the clinician's role in taking a careful case history and in modifying treatment when necessary throughout care.
\end{abstract}

Keywords: Shield ulcer, giant papillae, superficial keratopathy, supratarsal injection, local concoction.

\section{Résumé}

La kératoconjonctivite vernale (KCV) est une inflammation allergique, bilatérale et récurrente de la conjonctive et de la cornée dont l'incidence est saisonnière. Elle sévit plus chez les hommes que chez les femmes dans la tranche d'âge trois à seize ans chez les jeunes. Dans la majorité des cas, les symptômes disparaissent à la puberté, mais dans certains cas, ils peuvent persister jusqu'à l'âge adulte. Le diagnostic repose normalement sur les signes et symptômes cliniques. La cause de cette affection n'est pas clairement établie, mais elle se trouve souvent liée à des affections atopiques comme l'asthme ou l'eczéma et est probablement imputable à une réaction allergique de vieille date. Elle est cliniquement des types palpébral, limbique et mixte. Sa prise en charge a présenté tout un défi pour les soignants oculovisuels à cause de son caractère récurrent, du grand nombre de sujets touchés, des erreurs de diagnostic et de l'absence de posologie judicieuse. L'affection risque d'engendrer de graves complications qui viendront menacer la vue si la maladie n'est pas convenablement prise en charge. Dans cette étude de cas, nous passerons aussi en revue les options de diagnostic et de gestion dans le cas des patients atteints d'une KCV du type mixte et nous démontrerons l'importance du rôle du clinicien qui doit relever soigneusement les antécédents et modifier le traitement si nécessaire tout au long des soins.

Mots clés : Ulcère de la cornée, papilles géantes, kératite superficielle, injection supratarsienne, préparation locale. 


\section{Introduction}

Allergic conjunctivitis is the inflammation of the conjunctiva due to allergens. The inflammation caused by the allergens may be seasonal and involve both the conjunctiva and the cornea, hence the name vernal keratoconjunctivitis (VKC). Other clinical forms are included in the classification of ocular allergy from which VKC must be distinguished: seasonal/intermittent allergic conjunctivitis (SAC), perennial/persistent allergic conjunctivitis (PAC), atopic keratoconjunctivitis (AKC), giant papillary conjunctivitis (GPC) and drug-induced dermatoconjunctivitis. Amongst these, AKC is closest in appearance to VKC. ${ }^{1,2,17}$

This review focuses on the management challenges of VKC, a seasonal, recurrent, bilateral, chronic allergic inflammation of the conjunctiva and the cornea. ${ }^{2,6}$ The allergic response is triggered by an over-reaction of the body's immune system to allergens or foreign bodies and tends to run a more severe course.

It is also known as 'seasonal conjunctivitis,' 'spring catarrh' or 'warm weather conjunctivitis.. . It is the only ocular disease to involve solely Type 1 hypersensitivity response mediated by IgE antibodies bound to the membrane of the mast cell in the conjunctival substantia propria., ${ }^{3,14}$ Attachment of the antigen to the IgE antibodies results in the release of pro-inflammatory mediators like histamines, leukotrienes, prostaglandins, tryptase, chymase, platelet activating factor and other chemoattractants. This results in increased vascular permeability and attraction of eosinophils and neutrophils. ${ }^{3,7,12}$

These pro-inflammatory mediators are responsible for the clinical pictures of the conjunctivitis. ${ }^{3}$ However, cytologic, biohumoral, immunohistologic and molecular biologic studies have suggested the involvement of $\mathrm{T}$ helper cell type 2 (Th2) lymphocyte mediated allergic reaction with additional hypersensitivity responses that are often ill-defined. ${ }^{7,12,13}$ Vernal keratoconjunctivitis is usually bilateral, but may occasionally be asymmetrical. It accounts for roughly 0.10 to $0.50 \%$ of patients with ocular diseases worldwide. ${ }^{8,18}$ However, unilateral $\mathrm{VKC}$ is rare.

The disease affects children between 3 to 16 years of age, though it may appear earlier than that and continue into adulthood. In some cases, it is often self-limiting and the symptoms resolve around puberty. ${ }^{6}$ It is more common among boys than in girls probably due to their persistent outdoor activities. ${ }^{1}$ Its predominance in male children, resolution at puberty and positive staining for oestrogen and progesterone receptors in conjunctiva of patients with VKC also suggest a hormonal component in the development of VKC. ${ }^{7,9,16}$ In addition, increased serum levels of interleukin-17
(IL-17) and antinuclear antibodies, together with a high association with familial history of autoimmune disorders suggest additional mechanisms involved in the development of VKC. ${ }^{7,11,13}$ Multiple factors can contribute to the aetiology of VKC including environmental allergens, climate and genetic predisposition. ${ }^{7}$ However, the suggestion of a hereditary association still remains unclear as no clear direct correlation with specific genetic loci has been made. ${ }^{5}$ The disease is minimal in temperate climates compared to warm climates, and almost nonexistent in cold climates. It is severe in spring, summer and incidence falls in the winter. It is more common in Sub-Saharan Africa and the Middle East. ${ }^{1}$

Though the name vernal implies a seasonal occurrence, the disease may be perennial (persisting throughout the year) or with acute exacerbations. ${ }^{18}$ Severe VKC greatly affects a child's academic performance and ultimately impairs his quality of life. The corneal involvement in the disease known as vernal keratitis may be severe enough to interrupt a child's education and ultimately affect his future potential. Besides, it can lead to permanent visual impairment if not adequately managed. ${ }^{15}$ Vernal keratitis is characterized by a combination of punctate epithelial erosions and keratitis. ${ }^{3}$ Punctate epithelial erosions are areas of absent epithelium that stain with fluorescein. Punctate epithelial keratitis is caused by an influx of white cells into the corneal epithelium. The epithelium in this area stains with Rose Bengal, which stains devitalized cells. ${ }^{2,9}$ Keratitis epithelialis of Tobgy is the early phase of vernal keratitis, consisting of minute white dots in the epithelium which when coalesce, forming vernal ulcers known as shield ulcers., ${ }^{716}$ Shield ulcers are distinctly indolent, oval in shape and most often found in the upper half of the cornea. The pathogenesis of these shield ulcers is believed to involve a combination of mechanical damage to the corneal epithelium by giant papillae and toxic epitheliopathy from inflammatory mediators secreted by eosinophils and mast cells.,15 These ulcers are sterile but may, by chance, be infiltrated by bacterial pathogens giving rise to bacterial corneal ulcers. These ulcers can be differentiated from vernal shield ulcers, which are indolent and often have a plaque at the base. Shield ulcers occur in approximately $3 \%$ to $20 \%$ of patients with VKC. ${ }^{7,15,16}$

\section{Causes}

The aetiology and pathophysiology of VKC remain unclear, but may be due to a longstanding allergic reaction of the body's immune system to allergens. ${ }^{4}$ It is common in people who have other signs of allergic diseases such as hay fever, asthma, and eczema. ${ }^{4,6}$ Prominent causative allergens include pollen from trees, grasses and ragweed; animal dander; dust; smoke; 
mould spores; hairs; dust mites; wool and feathers; perfumes; cosmetics; eye drops; and skin medicines. ${ }^{1,8}$

\section{Symptoms}

The symptoms are due to the release of histamines and other active substances by the mast cells, which stimulate dilation of blood vessels, irritate nerve endings and increase secretion of tears.

Common symptoms include severe itching, tearing, photophobia (sensitivity to light), pain, eyelid swelling, irritation, blepharospasm, burning and foreign body sensation. Blurring of vision may occur when the cornea is involved. These complaints, especially itching, are made worse when the patient is exposed to a dusty, warm humid atmosphere, wind and bright light, or physical exertion associated with sweating, possibly due to increase in allergen count. ${ }^{3,6,8,16}$

\section{Signs}

The disease is characterized by milky appearance of the conjunctiva, fine papillae in the lower tarsal conjunctiva and giant, flat-topped papillae resembling a 'cobblestones' in the superior tarsal conjunctiva (which is the hallmark of the disease). ${ }^{1,7}$ Tenacious mucus may form and adhere to the cobblestones. The conjunctiva may be swollen and hyperaemic. Thick, ropy strands of mucus or filamentary keratitis are present (mainly in severe VKC). ${ }^{16}$ Microscopic examination of the conjunctival biopsies reveal large numbers of scattered eosinophils and eosinophilic granules but these are less common on conjunctival smears. Basophils may also be present in the biopsy but not in the same proportion as eosinophils. High levels of proteins secreted by eosinophils (e.g. eosinophil cationic protein) have been found in the tears of VKC patients as well as atopic and allergic conjunctivitis. There are also low proportions of mononuclear and polymorphonuclear inflammatory cells in the exudates. ${ }^{1}$

Clinically, vernal keratoconjunctivitis consists of the palpebral, the limbal and the mixed types. ${ }^{3,6}$

The palpebral form of VKC is characterised by giant, flattopped papillae on the superior tarsal conjunctiva that gives a "cobblestones" appearance. ${ }^{6}$ Giant papillae consist of papillary conjunctival masses greater than $1 \mathrm{~mm}$ in size on the upper tarsal conjunctiva, with a proliferation of collagen underneath the conjunctival epithelium. Its mechanism of formation is mainly epithelial thickening and fibroblast proliferation., The presence of giant papillae signifies prolonged chronic inflammation. It may also indicate poor prognosis. The palpebral VKC is also characterized by sticky exudates and tightly packed papillae in the active phase of the disease which get more separated as the inflammation abates. Approximately $50 \%$ of the patients with the palpebral form of VKC have corneal involvement which may range from superficial punctate keratopathy to well-demarcated sterile corneal shield-like ulcer (frank epithelial loss) located superiorly. ${ }^{6}$ This may heal leaving behind a vascularised scar. Frank epithelial loss (shield ulcer) results from the abrasion of the underlying cornea from the keratinised epithelium of the giant papillae. It also gives rise to the complaint of foreign body sensation. ${ }^{11} \mathrm{~A}$ fine fibrinous pseudomembrane that is enhanced by heat may accumulate on giant papillae and is known as Maxwell-Lyon's sign. ${ }^{1}$ (A pseudomembrane is an aggregate of coagulated exudates that is attached to an inflamed conjunctiva which when removed leaves behind an intact epithelium). A pseudoptosis, mostly unilateral, may be noted. The cause is quite unclear, but could be as a result of palpebral thickening, heavy giant papillae, chronic eye rubbing or inflammatory insult to the levator palpebrae superioris muscle.

Limbal VKC is prominent in black races and females. ${ }^{6}$ It has a better prognosis. 3 The conjunctiva is thick, oedematous and hyperaemic. The papillae in this form are found at the limbus (corneal-scleral junction) mainly superiorly. At the top of each papilla are gelatinous white clumps of degenerated epithelial cells and eosinophils, called Horner-Trantas dots, seen mainly during the active phase of the disease. ${ }^{1,6}$ The conjunctival changes include hyperpigmentation, subconjunctival fibrosis, keratinisation and symblepharon. A pseudogerontoxon (arcus senilis-like haze) is often noted in the cornea adjacent to the limbal papillae. ${ }^{1,16}$ It is often seen as a waxing and waning grey-white lipid deposit in the peripheral, superficial stroma. Pseudogerontoxon is clinically important because it is an indication of previous allergic eye disease. Papillae can be differentiated from follicles by their red centres which consist of dilated blood vessels at the core of the papillae, surrounded by inflammatory cells including lymphocytes, plasma cells and eosinophils. Papillae consist of hyperplastic conjunctival epithelium thrown into numerous folds and can only develop in the palpebral and bulbar conjunctiva at the corneo-scleral junction (limbus). Follicles, on the other hand, are most prominent in the forniceal conjunctiva and consist of hyperplasia of lymphoid tissue within the stroma. They are clinically seen as multiple, discrete, slightly elevated lesions reminiscent of small grains of rice surrounded by tiny blood vessels. ${ }^{3}$ Follicles are commonly seen in viral, chlamydial infections, Parinaud oculoglandular syndrome and hypersensitivity to topical medications.

The mixed form of VKC has the characteristic features of both the palpebral and the limbal forms. 
Micropannus is often common in both palpebral and limbal VKC, but gross pannus is unusual. ${ }^{1}$ Neovascularisation of the cornea may follow. ${ }^{6}$ Conjunctival scarring usually does not occur unless the patient is treated with cryotherapy, surgical removal of papillae, irradiation or other damaging procedures. ${ }^{1}$ Impairment of vision is common in cases with shield ulcers and corneal plaques. The disease may be associated with ectatic corneal diseases such as pellucid marginal degeneration and keratoconus. ${ }^{1,3}$ Amblyopia seen among patients with VKC may be caused by corneal opacity, irregular astigmatism and keratoconus. ${ }^{16}$

The palpebral form of VKC is more prevalent In Europe and the Americas, whereas the limbal and the mixed forms are common in Africa and Asia respectively, with some geographical variation. ${ }^{5,7}$

\section{Challenges}

Allergic eye diseases and vernal keratoconjunctivitis, in particular, have been a thorn in the flesh of eye care providers and patients in warm climates. This ugly scenario has been attributed to the large percentage of the populace affected by the disease. ${ }^{6}$ Khaksar and Bagheri, in an epidemiological study of VKC in Kashan (in Iran), stated that approximately 3\% of the patients seeking eye care services in outpatient clinic of the Ophthalmology Department BVH, Bahawalpur had VKC and more than $80 \%$ are below 18 years of age. ${ }^{10}$ Also, Hall and Shilio of the Ophthalmology Department, Kilimanjaro Christian Medical Centre, Moshi, Tanzania stated that over $25 \%$ of the total children seen at a tertiary referral paediatric eye clinic in East Africa had VKC and more of the children came flocking to screening clinics complaining of itchy eyes. ${ }^{6}$

The unstable and unpredictable global weather conditions have increased the vulnerability of affected individuals who are unable to predict effectively exposure periods and take adequate precautionary measures.

The management is time-consuming and frustrating both to the patients and the eye care providers, in that patients keep coming with the same complaint often without appreciable remedy. ${ }^{6}$ The patients often seek different eye clinics and medicine dealers in search of cure when symptoms become unbearable. Sometimes, they may have suffered the affliction many years before being properly diagnosed.

The morbidity rate of blindness from the disease is high. Some patients that develop corneal ulcers may go blind due to corneal opacification. ${ }^{6}$ Other visual impairments such as glaucoma, dry eye syndrome, or cataract may result from uncontrolled use of steroids. ${ }^{16}$

Some continents like Sub-Saharan Africa and the Middle
East, where the disease is more prevalent, are populated by individuals living in abject poverty and unhygienic environment. The high costs of the potent topical medications for the disease are beyond the means of the populace. This compounds the problem, leaving the patients with no option but resort to the use of local concoctions, which may give rise to diverse ocular complications and blindness. On the other hand, some of these potent ocular medications are not readily available. When available, some are adulterated, thereby making disease response inadequate. The available effective drugs may not be judiciously used by some patients as prescribed, thus limiting management.

Inadequate man power to cater for the ever-increasing number of patients with ocular allergy is another problem. This has led to patronization of quacks with the resultant effect of visual impairment.

It is often said that he who is not informed is deformed. Lack of awareness of the disease by the patient, and the inability of some eye care providers to provide the patients with firsthand information of the nature, course and predisposing factors associated with their ailment constitutes another setback. This leaves the patient with the option of seeking information from the wrong channel which may even aggravate their condition.

The lack of standardized diagnostic criteria, absence of an established gold standard treatment, and lack of common language among physicians regarding the severity of VKC renders the disease more difficult to diagnose and treat. ${ }^{16}$

In order to tackle these challenges directly, eye care providers should have first-hand information of the nature of the disease, recognition of its clinical features and potential blinding complications. ${ }^{6}$

Given the chronic nature of the disease, regular and adequate treatment through proper patient education regarding what to expect and the pit falls of therapy as well as long term follow-up is essential. ${ }^{18}$ This can be backed up by patient information leaflets as a way of breaking the cycle of inadequate treatment, misuse of medication, noncompliance, and resulting frustration. ${ }^{6}$ Discouragement of self-medication through effective enlightenment campaigns by eye care providers and provision of potent low cost ocular medications will go a long way toward ameliorating the problem. However, psychological support may be necessary in severe cases.

Training and re-training of eye care professionals is of utmost importance to equip them with the latest developments in eye care profession. The use of community health care providers in reaching out to the vast populace in the rural areas far from health facilities should be encouraged. The public should be advised about the benefits of personal and 
environmental hygiene. Government and non-governmental organizations should collaborate in bringing heath care resources closer to the people for prompt and effective dispensing of treatment.

Medical outreach programmes can be organized by the eye care professionals. Policy makers should endeavour to incorporate physical and health education in the school curriculum from primary to tertiary level to raise awareness of common ocular and other diseases.

Government and non-governmental agencies should support medical research institutes and researchers with readily available funds and research grants. This will permit in-depth study of the disease and other ailments, with the aim of establishing standardized diagnostic criteria and a universally acceptable model of treatment.

\section{Treatment}

Vernal keratoconjunctivitis treatment involves alleviating the severity of the symptoms and preventing serious sight threatening complications. Permanent visual impairment can result from improper management of the disease.

The first step in the management of vernal keratoconjunctivitis is patient counselling. This is aimed at educating the patients on the nature, course and predisposing factors associated with the diseases. ${ }^{6}$ This will go a long way toward good adherence to the symptomatic management and avoidance of those environmental factors that may exacerbate the disease.

Topical mast cell stabilizers such as Alomide (Lodoxamide Tromethamine 0.1\%), Alocril (Nedocromil Sodium 2\%), and Crolom (Cromolyn sodium 4\%) can be given in mild to moderate cases. They should be used regularly 3-4 times daily even when the symptoms have subsided to prevent the release of histamines from the mast cells. However, their use after the symptoms have occurred is of no value because their action is not immediate. They can be used for a long time, for they do not have the side effects of steroids. They are not usually adequate when the eyes are severely inflamed, when keratitis is present, or when there is a vernal ulcer. In these cases, topical steroids should be used to control the inflammation and mast cell stabilizers added as adjunctive therapy to give room for steroid tapering. ${ }^{3,5,6,7}$

Topical mast cell stabilizing- antihistamines such as livostin (Levocabastine hydrochloride $0.05 \%$ ), patanol (Olapadine Hydrochloride 0.1\%), zaditor (Ketotifen Fumarate 0.025\%) and optivar (azelastine $0.05 \%$ ) are good in managing mild to moderate cases. ${ }^{114}$ They act by reducing eosinophil activation and cytokine release. ${ }^{1}$ Oral antihistamines like Benadryl $25 \mathrm{mg}$ three times daily are also effective in mild to moderate cases in suppressing other symptoms of allergy but are of limited benefit in the eye. ${ }^{3}$

Topical decongestants such as epinephrine, naphazoline, and phenylephrine may play an effective role in mild to moderate VKC by producing vasoconstriction of superficial vessels, reducing hyperaemia, chemosis and other symptoms and by retarding the release of chemical mediators. ${ }^{11,17}$ Cold compresses, ice packs, preservative free artificial tears and ointments may play a helpful role in diluting the antigens, thereby reducing the severity of the symptoms, but are irrelevant in managing onset of keratitis. Sleeping (if possible also working) in a cool, air conditioned room can keep the patient reasonably comfortable. Probably the best remedy of all is to move to a cool moist climate. ${ }^{1,8}$

Topical non-steroidal anti-inflammatory drugs (NSAID) such as Acular (Keteorolac Tromethamine 0.5\%), Diclofenac (voltaren) may provide significant symptomatic relief in moderate cases, but may retard the re-epithelialization of a shield ulcer. ${ }^{16}$ These agents act primarily by blocking the synthesis/release of prostaglandin through inhibition of cyclooxygenase, the enzyme that catalyses the conversion of arachidonic acid to prostaglandins. ${ }^{1}$ However, a careful follow-up is required as corneal melting has been reported after instillation of several types of NSAID. ${ }^{5}$

A short term course of systemic and or topical steroids such as Pred Forte, Lotemax (loteprednol etabonate 0.5\%), Alrex or Pred Mild (prednisolone 0.12\%, Allergan) and Efemoline followed by cold packs and histamine blocking agents can be used in severe cases as well as in those presenting with corneal involvement. ${ }^{7,14}$

Steroids should be avoided as the first line of defence in the treatment of mild to moderate VKC because of their long term side effects. ${ }^{16}$ In using steroids, those with low intraocular absorption such as Fluorometholne, Lotoprednol, or Efemoline should be used first as they have less or no effect in intraocular pressure (IOP). Stronger steroids such as Prednisolone, Dexamethasone, or betamethasone should be used with adequate supervision only when the abovementioned first choice steroids have proven ineffective.

Steroids inhibit mediator biosynthesis and disrupt intercellular communication by preventing the release of lymphokines. They are the most effective and best proved treatment for VKC especially in active keratitis. ${ }^{5,17}$

Steroids should be used frequently initially and tapered to a stop once the acute stage of the disease is stabilized (usually a few weeks). Prolonged use of steroids should be avoided since it is too often followed by herpes simplex keratitis, increase 
in IOP, cataract, glaucoma, delayed wound healing, dry eye syndrome, fungal and other opportunistic corneal ulcers. ${ }^{6,9,16}$

Supratarsal injections of both long acting steroids (e.g. Triamcinolone acetonide), and short acting steroids (e.g. Dexamethasone sodium succinate) may be used for those not responding to conventional treatment. ${ }^{6,10}$

Steroid-antibiotic combination eye drops should be avoided as VKC is an allergic inflammation rather than an infection. In cases of secondary bacterial infection, topical antibiotic eye drops and/or ointment should be used to provide adequate therapeutic dose to combat the infection.

Topical Cyclosporine A (CsA) drops (1-5\%) e.g. Restasis, in olive oil or castor oil 4 times a day can be used as an alternative to steroid injection in severe cases. ${ }^{6,7,12}$ Cyclosporin $\mathrm{A}$ is a calcineurin inhibitor that binds to cyclophilin, an intracellular protein, which in turn prevents the formation of interleukin-2 and the subsequent recruitment of activated $\mathrm{T}$ cells. It also inhibits histamine release from mast cells. Through reduction of IL-5 production it may reduce the recruitment and the effect of eosinophils on the conjunctiva. ${ }^{12,16,19}$ It has been used successfully in several studies to treat severe cases of VKC. It does not cause an increase in IOP. It is a potent immunosuppressive agent used in the prevention of transplant rejections. It plays an important role in the healing of vernal shield ulcers but recurrences may occur at lower concentrations, which may mandate increasing the concentration. Its use in VKC management is limited due to its high cost and unavailability of commercial preparation of topical CsA in higher concentrations. ${ }^{13,16,19}$

Tacrolimus is a potent drug, similar to CsA in its mode of action, but chemically distinct. Case series and placebo controlled randomized control clinical trial has shown a beneficial effect of tacrolimus ointment $(0.1 \%$ to $0.3 \%)$ and drops $(0.005 \%$ and $0.1 \%)$ in severe VKC. However, it irritates the eyes and makes patients susceptible to opportunistic infections and herpes simplex keratitis. It has a limited use in the developing world due to its high cost. ${ }^{21}$

Tenacious mucus adhering to the cobblestones in particular, thick, ropy strands of mucus as well as filamentary keratitis can be treated with Acetylcysteine. It is known to break the disulfide bonds, thereby dissolving the mucus. ${ }^{16}$ It is formulated from commercially available Mucomyst, diluted to a $5 \%$ or $10 \%$ solution with artificial tears, and is applied four times a day. It is also effective in treatment of shield ulcer in conjunction with aggressive cycloplegia (homatropine 5\% or scopolamine $0.25 \%$, bid) and topical antibiotic dropsCiloxan (ciprofloxacin, Alcon); Ocuflox (ofloxacin, Allergan) or Quixin (levofloxacin, Santen). ${ }^{11,16,19}$
Short-term, low-dose, topical mitomycin-C $0.01 \%$ has been considered for treating acute exacerbations in patients with severe VKC refractory to conventional treatment. It inhibits fibroblast proliferation and decreases mucus discharge, conjunctival hyperaemia and limbal oedema. However, unavailability of commercial topical preparations, limited comparative studies, and the lack of data on the safety profile and long-term outcomes are major limitations in recommending mitomycin for the treatment of VKC. ${ }^{16,20}$

Debridement/lamellar keratectomy of early mucus plaques at the base of the vernal ulcer and intensive topical steroids may speed up the re-epithelialization process. Also, low-watercontent bandage contact lenses are helpful in the treatment of these defects as well as reducing the interaction between the lid and cornea. ${ }^{5,6,16}$

Free autologous conjunctival graft resection of giant papillae facilitates the re-epithelialization of non-healing shield ulcer. Persistent corneal epithelial defects can be treated by amniotic membrane transplantation and limbal epithelial cell transplantation. Vision improves significantly after transplant. Corneal epithelial cell transplants could be beneficial when amniotic membrane transplant is not sufficient to restore the ocular surface. ${ }^{3,12,16}$

Other general measures in the management of VKC include: dark goggles (sunglasses), visors and caps to prevent photophobia. Identification of the non-specific triggering agents which could aggravate symptoms such as strong wind, dust, air pollutants and strong sunlight and avoiding them are of optimum importance but this is often impractical. Frequent hand and face washing with clean cool water or rinsing the eye with an adequate amount of cool normal saline is also important to reduce the severity of the symptoms and avoid secondary infection.

\section{Case Presentation}

A 13-year-old male patient presented to the clinic with complaints of severe itching, discharge, blurry distance vision with object distortion and discomfort with light in both eyes. The problem started about two and a half months earlier and he had been on chloramphenicol eye drops to no avail. Further probing into his case revealed that his condition was usually made worse whenever he visited their poultry house. He had been known to have seasonal allergies right from birth and had never had an eye examination before. There is also a family history of eczema.

Clinical examination revealed the following: unaided visual acuity OD: $20 / 60$ (6/18), PH: $20 / 40^{-2}\left(6 / 12^{-2}\right)$. OS: $20 / 60^{+2}\left(6 / 18^{+2}\right)$, PH: $20 / 40(6 / 12)$. The patient was moderately 
photophobic with no relative afferent papillary defect (RAPD) in both eyes. The intraocular pressure (IOP) was OD: $16 \mathrm{mmHg}$ and OS: $17 \mathrm{mmHg}$. Slit lamp examination revealed dense papillary reaction with some thick strands of mucus adhering on the palpebral surface of the upper lids. There was also moderate papillary reaction of the limbal conjunctiva superiorly and a dark brownish colouration of the conjunctiva around the limbus, more profound at the palpebral fissure in both eyes. Dilated fundus examination revealed normal disc, macula, vessels and peripheral fundus in both eyes. Refraction before dilated funduscopy gave the following results.

\section{Objective refraction (Autorefractor):}

OD: +0.25 - 3.50 x 045 VA 20/40 (6/12); OS: +0.75-5.50 x 130 VA $20 / 40^{+2}\left(6 / 12^{+2}\right)$

\section{Subjective refraction:}

OD: $\mathrm{Pl}-2.00$ x 15 VA 20/40 (6/12); OS: +0.25 - 3.50 x 115 VA $20 / 40^{+2}\left(6 / 12^{+2}\right)$

The patient was diagnosed with mixed vernal keratoconjunctivitis; animal danders were suspected as the triggering agent. He was started on topical steroid Lotemax (Loteprednol etabonate $0.5 \%$ ) twice daily due to the severity of the disease and topical antihistamine/mast cell stabilizerZaditor (Ketotifen fumarate $0.025 \%$ ) twice daily. He was advised against visiting their poultry house or other animal farms outside their home, coming in contact with pets, and to discontinue the application of chloramphenicol eye drops. He was also advised on the need for proper personal hygiene, regular washing of hands and face with cool clean water, scrubbing of the eyelash with cotton tipped applicator soaked in normal saline to remove the dry exudates. The subjective refraction result was not dispensed due to the suspected compromise in the topography of the corneal surface due to the active nature of the disease. On his follow-up visit two weeks later, there was an improvement of the limbal papillae but the superior tarsal conjunctival papillae persisted together with the dark limbal colouration. He was advised to continue with the two drugs and to return to clinic after one week. On his next visit one week later, there was a complete resolution of the limbal papillae and some resolution of the dark limbal colouration but the superior tarsal papillae persisted with only moderate clearance of mucus adherence. He was then given a supratarsal injection of $0.5 \mathrm{ml}$ triamcinolone under local anaesthetic drops as follows: The conjunctiva was anaesthetized with $4 \%$ lidocaine hydrochloride drops instilled to both eyes every minute for five minutes, after the course of treatment has been explained and his consent obtained. The upper eyelid was everted. $4 \%$ lidocaine hydrochloride soaked cotton tipped applicator was dabbed over it for about 1 minute. The patient was asked to look down and a 26 gauge needle of $1 \mathrm{ml}$ syringe containing $0.5 \mathrm{ml}$ of triamcinolone acetonide $(40 \mathrm{mg} / \mathrm{ml})$ was inserted through the conjunctiva into the supratarsal space between the conjunctiva and the Mueller's muscle. Adequate care was taken to avoid the marginal blood vessels. $0.5 \mathrm{ml}$ of triamcinolone acetonide was injected after ensuring proper placement of the needle. The potential space between the conjunctiva and Mueller's muscle was ballooned indicating successful placement of the injection. Both eyes were injected and a pressure pad was applied for 2 to 3 minutes to reduce the ballooning of the lid and help spread the injection evenly. He was maintained on Zaditor after the injection but discontinued topical steroid after tapering it one drop daily for 1 week. On his next visit 2 weeks later there was a total resolution of the papillae and the limbal brownish colouration could hardly be noticed. There was no significant increase in the IOP as it stood at $17 \mathrm{mmHg}$ in both eyes. His final optical prescription after resolution was OD: $\mathrm{Pl}-1.25 \times 35 \mathrm{VA} 20 / 30^{+2}\left(6 / 9^{+2}\right)$ and OS: $\mathrm{Pl}-2.00 \times 145 \mathrm{VA} 20 / 20^{-3}\left(6 / 6^{-3}\right)$ with VA in both eyes as $20 / 20^{-2}\left(6 / 6^{-2}\right)$. The spectacle prescription was dispensed to him in photochromic form to enhance his vision (as well as eliminating image distortion) and reduce the effect of light. He was placed on regular use of Zaditor to avoid sudden reoccurrence. He was counselled on the nature of his disease and the need to avoid coming in contact with the suspected predisposing agent and the importance of regular clinical checks despite resolution.

\section{Discussion}

Vernal keratoconjunctivitis is a seasonal, bilateral, recurrent allergic inflammation of the conjunctiva and the cornea. ${ }^{2,6}$ The disease affects children between 3 and years of age, though it may appear earlier than that and continue into adulthood. In some cases, it is often self-limiting and the symptoms resolve around puberty. ${ }^{6}$ It is more common among males than females. ${ }^{1}$ It is characterized by the presence of bilateral palpebral and/or bulbar conjunctival papillae, photophobia, tearing, burning, thick, ropy, yellow mucoid discharge and mild to severe itching. ${ }^{3,6,7}$ It has a high incidence in the warm, dry climates with fewer incidences in cooler climates. ${ }^{1}$ It is associated with a positive family history for atopic diseases such as asthma, rhinitis, hay fever and eczema. ${ }^{4,6,7}$ In VKC, an allergic response is triggered by an over-reaction of the body's immune system to allergy or foreign bodies. This leads to the release of histamines and other mediators by the immune 
system resulting in itching, burning and runny eyes that may become red and inflamed. Classically, it is thought of as a type $1 \mathrm{IgE}$-mediated hypersensitivity reaction. However, it has been suggested that there is a cell-mediated Th-2 involvement. ${ }^{3,7,13}$ Laboratory testing of the conjunctival scrapings reveals higher numbers of eosinophils. ${ }^{1}$ Finding eosinophilic granules can aid the diagnosis but is not necessary, except in difficult cases, as VKC is easily diagnosed clinically based on history, signs and symptoms. ${ }^{14,16}$

The main idea in the management of VKC is to minimize inflammation as well as to reduce complications until the disease subsides. Topical treatment is preferable and more effective than systemic. Topical mast cell stabilizers and antihistamines or a combination of both are the first line of choice in the management. ${ }^{14,16}$ The drugs are effective for mild to moderate cases and can be used for a long period without adverse effect. Severe and unresponsive cases are treated with steroids and cyclosporine. Non-steroidal anti-inflammatory eye drops are also useful in mild cases. However, detection and avoidance of the triggering agents (allergens), cold compresses, and moving to a cooler climate is a good strategy in ameliorating the severity of the disease. . $^{1,6,14}$

In this case, the blurry distance vision resulted from astigmatism brought about by the compromise of the corneal surface due to the activeness of the disease. (This manifests from the discharge, limbal papillae, tarsal palpebral papillae pressing on the eyeball and constant rubbing of the eye due to intense itching which may slightly alter the topography of the cornea). The photophobia results from the painful contraction of an inflamed iris or the excitation of the many pain fibres of the cornea. The discharge comes from the migration of inflammatory cells (e.g. eosinophils) from the conjunctival stroma through the epithelium to the surface. Here they combine with fibrin and mucus from the goblets cells to form the conjunctival exudates ${ }^{1}$ Topical mast cell stabilizer (Zaditor) and topical steroid (Lotemax) were prescribed due to the severity of the disease. These produced a good response in resolving the limbal component of the disease. The unresponsive tarsal palpebral component was taken care of by supratarsal injection of $0.5 \mathrm{~mL}$ triamcinolone acetonide which gave a complete resolution and disappearance of the brownish colouration. The Zaditor was continued after the resolution mainly to avoid a relapse. There was no significant change in the IOP of the patient due to adequate monitoring and close supervision of the drug administration. The refraction result obtained before the resolution of the disease was not dispensed due to its unreliability brought about by the compromise of the corneal surface as a result of the active nature of the disease. However, the result obtained after complete resolution which gave a significant visual improvement was dispensed in photochromic form to reduce the patient's sensitivity to light. Eliminating exposure to the identified triggering agent (animal dander) accelerated the early resolution and should prevent recurrence.

Differential diagnosis includes giant papillary conjunctivitis due to retained foreign body, exposed sutures and contact lens related problems, chlamydial and gonococcal conjunctivitis, chronic blepharitis, atopic keratoconjunctivitis, viral and toxic conjunctivitis, SAC, PAC, phlyctenulosis, band keratopathy and floppy eyelid syndrome. ${ }^{2,8,14}$ This is possible through a comprehensive clinical history and ocular examination. AKC rarely occurs in childhood and patients tend to complain of a watery discharge (mucus discharge in VKC).

Conjunctival scraping reveals eosinophils to a greater degree in $\mathrm{VKC}$, the inferior palpebral conjunctiva is more involved (usually superior in VKC), and there is often an associated blepharitis (less likely in VKC). ${ }^{1,3}$ Differentiating VKC from the other allergic conditions is usually fairly straightforward: corneal changes are rarely seen in SAC and PAC; in GPC, the ocular irritation is associated with contact lens wear; and in drug induced dermato-conjunctivitis, the offending agent can usually be identified. Symptoms of infective conjunctivitis are normally worse first thing in the morning and the eyelids are often gummed together on awakening due to discharge. In viral conjunctivitis, small conjunctival haemorrhages are common. Tenderness and swelling of the preauricular nodes is indicative of viral conjunctivitis. Bacterial conjunctivitis is characterised by purulent discharge. ${ }^{1,4,17}$

In conclusion, since a gold standard treatment for VKC has not been established, understanding of the pathogenesis of the disease by eye care providers may lead to better therapy for the patients with this unfortunate disease.

\section{References}

1. Francisco JG, Ivan RS, Debra JS. Conjunctiva. In: Asbury T, Riordan-Eva P, Vaughan T. eds. General Ophthalmology, 17th ed. New York: McGraw-Hill; 2007: 98-113.

2. Khurana AK. Comprehensive Ophthalmology, 4th ed. New Delhi: New Age International; 2008: 73-77.

3. Kanski JJ. Clinical Ophthalmology, 6th ed. Oxford UK: Butterworth \& Heinemann; 2007: 215-235.

4. Senaratne T, Gilbert C: Conjunctivitis. Comm. Eye Health J. 2005; 18(53):73-5.

5. Bonini S, Schiavone M, Centofanti M, et al: Vernal Keratoconjunctivitis revisited: a case of 195 patients with long-term follow-up. Ophthalmol. 2000; 107(6):1157-63.

6. Hall A, Shilio B: Vernal keratoconjunctivitis. Comm. Eye Health J. 2005; 18(53):76-8.

7. Bonin S, Coassin M, Aronni S, Lambiase A: Vernal keratoconjunctivitis. Eye.2004; 18:345-51. 
8. http://www.Kellogg.umich.edu/patientcare/condition/vernal html (last accessed 10/03/2015)

9. Wakefield D, McCluskey PJ: Vernal keratoconjunctivitis. The medical journal of Australia. 2006; 185(9):523-5.

10. Muhammad RQ, Ejaz L, Tariq MA, Ehsan U: Supratarsal injection of triamcinolone for vernal keratoconjunctivitis. Pak J Ophthalmol. 2010; 26((1):28-31.

11. Spaclavacchia L, Fanelli P, et al: Prognosis and treatment of vernal keratoconjunctiviti in paediatric age pilot study on 197 patients. Researchgate. 2010; 62(3):239-44.

12. Ben Ezra D, Pe'er J, Brodsky M, et al: Cyclosporin eye drops for the treatment of severe vernal keratoconjunctivitis. Am J Ophthalmol. 1986; 101:278-82.

13. Secchi AG, Tognon MS, Leonardi A: Topical use of cyclosporine in the treatment of vernal keratoconjunctivitis. Am J Ophthalmol. 1990; 110:641-5.

14. Koczman J, Oetting TA: Vernal Keratoconjunctivitis: 8 year-old asthmatic male with reduced vision. EyeRounds.org. June 25, 2007; Available from: http://www.EyeRounds.org/cases/70-Vernal-Keratoconjunctivitis-AtopicAsthma.htm.
15. Arun KJ, Jaspreet S, Ira C: Keratic precipitates in bilateral vernal corneal ulcer. Asian J Ophthalmol. 2006; 8(4):159-60.

16. Sunil k: Vernal keratoconjunctivitis: a major review. Acta Ophthalmologica. 2009; 87(2):133-47.

17. Allansmith MR: Vernal keratoconjunctivitis. Duane's Clinical Ophthalmology. 4th ed. Philadephia: Lippincot-raven; 1994: 1-8.

18. Suresha AR, Farhat F, Avinash S: A clinical study of vernal keratoconjunctivitis. International J Biomedical Research. 2014; 5(4): 284-7.

19. Utine CA, Stern M, Akpek EK: Clinical review: topical ophthalmic use of cyclosporine A. Ocul Immunol Inflamm. 2010; 18(5): 352-61.

20. Akpek EK, Hasiripi $\mathrm{H}$ et al: A randomised trial of low-dose, topical mitomycin-C in the treatment of severe vernal keratoconjunctivitis. Ophthalmol. 2010; 107(2): 263-9.

21. Rikkers SM, Holland GN, Drayton GE et al: Topical tacrolimus treatment of Atopic eyelid diseases. Am J Ophthalmol. 2003; 135(3): 297-302.

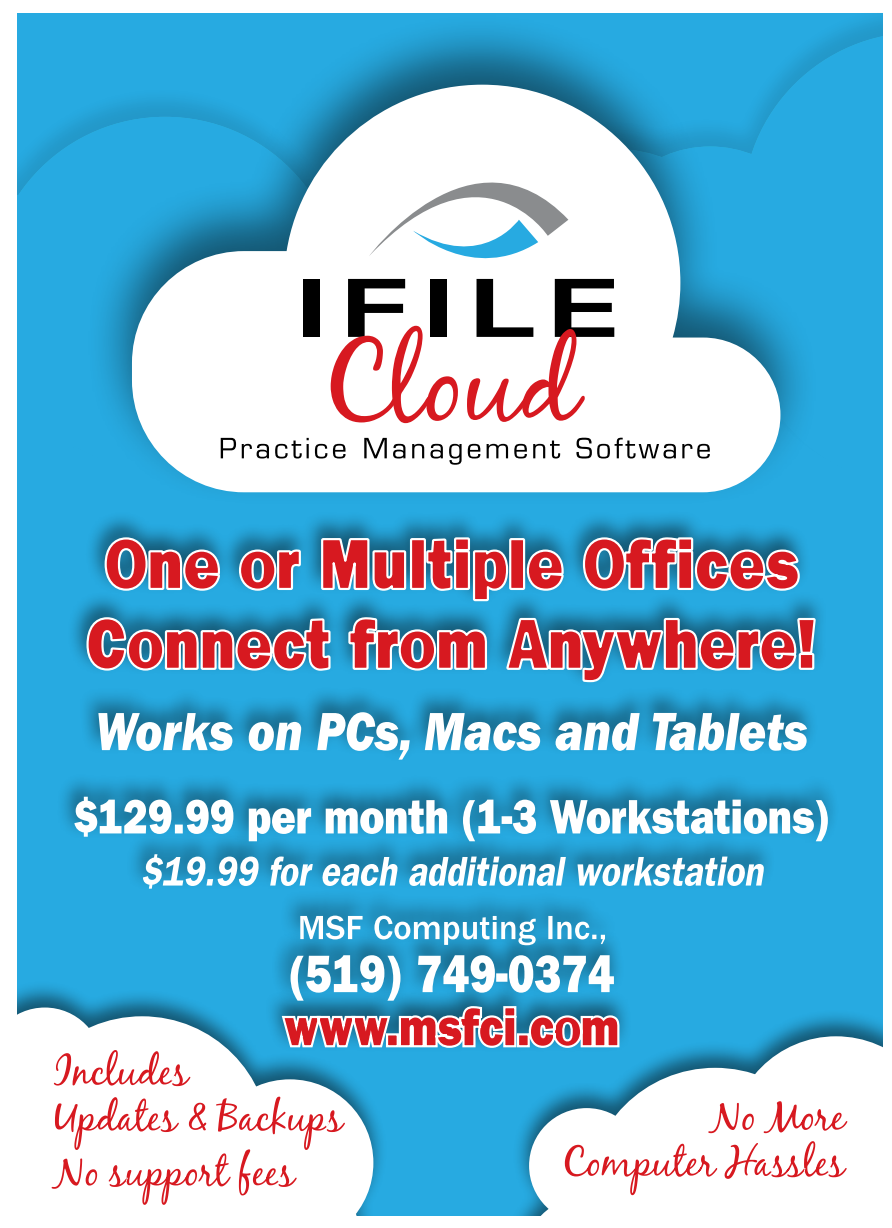

